\title{
Early Stage Clustering Behavior in Al-Mg-Si Alloys Observed via Time Dependent Magnetization
}

\author{
Katsuhiko Nishimura ${ }^{1}{ }^{*}$, Kenji Matsuda $^{1}$, Qiankun Lei ${ }^{1}$, Takahiro Namiki ${ }^{1}$, Seungwon Lee ${ }^{1}$, \\ Norio Nunomra ${ }^{2}$, Teiichiro Matsuzaki ${ }^{3}$ and Wayne D. Hutchison ${ }^{4}$ \\ ${ }^{1}$ Graduate School of Science and Engineering, University of Toyama, Gofuku, Toyama, 930-8555, Japan \\ ${ }^{2}$ Information Technology Center, University of Toyama, Gofuku, Toyama, 930-8555, Japan \\ ${ }^{3}$ RIKEN Nishina Center for Accelerator Based Science, RIKEN, Wako, Saitama 351-0198, Japan \\ ${ }^{4}$ School of Physical, Environmental and Mathematical Sciences, The University of New South Wales, Canberra ACT 2600, Australia
}

Time dependent magnetization of Al-0.67 at.\%Mg-0.73 at. $\% \mathrm{Si}, \mathrm{Al}-1.07$ at. $\% \mathrm{Mg}-0.33$ at. $\% \mathrm{Si}$ and $\mathrm{Al}-1.07$ at. $\% \mathrm{Mg}-0.53$ at. $\% \mathrm{Si}$ alloys are presented over a range of constant temperatures between 250 and $320 \mathrm{~K}$. The magnetization vs. time curves for the samples show minima for temperatures near $290 \mathrm{~K}$. The observed times at which the magnetization minima occur were found to depend on both the solute concentrations and the measurement temperatures. From these results the activation energies from the Si-rich clustering stage to the Mg-Si co-clustering stage were extracted. The deduced activation energies were found to be comparable to those from the positron annihilation measurements, depending on the solute concentrations. [doi:10.2320/matertrans.M2015474]

(Received December 24, 2015; Accepted February 9, 2016; Published March 18, 2016)

Keywords: time dependent magnetization, clustering reaction, activation energy

\section{Introduction}

Strong, light weight metals are essential to industry, especially for transport where high energy-efficiency is an important criterion. The Al-Mg-Si (6xxx series) aluminum alloys are in high demand as materials for vehicles because of their low weight, excellent formability and age hardenability. ${ }^{1,2)}$ The usual process for heat treatment is a solution heat treatment (SHT) at around $820 \mathrm{~K}$ followed by a quick quench to water, resulting in a supersaturated solid solution (SSSS). After inevitable storage at room temperature (called natural aging, NA), the alloy is given an artificial aging (AA) at around $420 \mathrm{~K}$, leading to a precipitation sequence: : $^{3,4)}$

$\mathrm{SSSS} \rightarrow \mathrm{Mg} / \mathrm{Si} /$ vacancy cluster $\rightarrow$ Guinier Preston (GP) zone $\rightarrow \beta^{\prime \prime} \rightarrow \beta$ ' $\rightarrow \beta\left(\mathrm{Mg}_{2} \mathrm{Si}\right)$.

It is well known that the early stage of solute clustering of $\mathrm{Si}$ and $\mathrm{Mg}$ proceeds quite quickly and often is completed in less than an hour even at room temperature. ${ }^{5-9)}$ A long-standing problem for industry is that some periods of NA often result in a negative effect on the mechanical hardness in the following AA. ${ }^{4,10-12)}$ The microstructures of the precipitations have been studied intensively via transmission electron microscopy $(\mathrm{TEM})^{13,14)}$ and atom probe tomography $(\mathrm{APT})^{3,15-17)}$ to reveal the age hardening mechanism. These techniques, however, require time consuming sample preparations, thus it is difficult to observe directly the early stage of clustering. Differential scanning calorimetry (DSC) ${ }^{18-21)}$ has been widely used to investigate the precipitation processes and cluster formations, but in principle this method cannot be used for isothermal measurements since peak positions in a heat flow spectrum depend on a heating rate. From the various studies with Al-Mg-Si alloys, vacancy behavior is considered to play an important role in the aging process, stimulating diffusion of solute $\mathrm{Mg}$ and $\mathrm{Si}$ atoms and nucleation of clusters. Positron annihilation spectroscopy (PAS) ${ }^{6-9)}$ and

*Corresponding author, E-mail: nishi@eng.u-toyama.ac.jp muon spin relaxation spectroscopy $(\mu \mathrm{SR})^{22-24)}$ have been successfully used to investigate the vacancy and clustering behavior in Al-Mg-Si alloys. These methods, however, are not widely accessible as special facilities and equipment are required to handle radioactive materials. Quite recently we have found that conventional DC magnetization of Al-1.07 at. $\% \mathrm{Mg}-0.53$ at. $\% \mathrm{Si}$ could provide similar information to the previous studies. ${ }^{25}$ )

In this paper we expand the studies, confirming that DC magnetization can track time development of the early stage clustering of $\mathrm{Mg}$ - or/and Si-vacancy in $\mathrm{Al}-\mathrm{Mg}$-Si alloys with different solute $\mathrm{Mg}$ and $\mathrm{Si}$ concentrations. Magnetization measurements were carried out for Al-0.67 at. $\% \mathrm{Mg}-0.73$ at. $\% \mathrm{Si}$ (noted as $\mathrm{Al}-7-7$ ) and $\mathrm{Al}-1.07$ at.\% $\mathrm{Mg}-0.33$ at. $\% \mathrm{Si}$ (Al-10-3) at a number of constant temperatures between 250 and $320 \mathrm{~K}$ (NA temperature) and over a time range from approximately 12 to 1300 minutes after SHT. A measurement on the Al-1.07 at.\%Mg-0.53 at.\%Si (Al-10-5), additional to the previous work, was carried out at $270 \mathrm{~K}$. The magnetization vs. time curves with these three samples showed minima at NA temperatures around $290 \mathrm{~K}$. In addition, the times of the magnetization minima were found to depend on the solute concentrations, as well as the NA temperatures. These results enable us to extract the activation energies for the clustering process, which can be compared with values reported previously.

\section{Experimental Procedure}

The experimental procedure was the same as that in our previous work. ${ }^{25)}$ Ingots of Al-7-7, Al-10-3 and Al-10-5 alloys were prepared by melting pure Al (99.99\% purity) with $\mathrm{Si}$ and $\mathrm{Mg}$ (purity 99.9\%) in air. The resulting ingots were formed into $2.5 \mathrm{~mm}$ thick plates by hot and cold rolling. Several pieces of the samples to be used for magnetization measurements were cut out from the plate with the approximate dimensions of $2.5 \times 2.5 \times 5.0 \mathrm{~mm}$. Prior to the magnetization 
measurements, samples were annealed at $848 \mathrm{~K}$ for 1 hour and directly quenched into ice-water (SHTQ). It took about 12 minutes to load the sample into a superconducting quantum interference device (SQUID) magnetometer (Quantum Design, MPMS-XL7), then apply an external magnetic field of 7 tesla (T) produced with a superconducting solenoid in a persistent mode and started the measurements.

\section{Results and Discussions}

The time variation of magnetization $(M)$ of the Al-7-7 sample at $290 \mathrm{~K}$ and $7 \mathrm{~T}$ is shown in Fig. 1. The horizontal axis denotes the time $(t)$ from SHTQ on a logarithmic scale. It is clear that $M$ varies with time with a minimum occurring at 55 minutes as marked by the arrow in Fig. 1. This magnetization minimum was estimated from the intersection of the two lines as shown. These lines are least-square fits to two adjacent parts of the data (on the logarithmic time scale), before and after the minimum, respectively. The observed $M$ vs. $t$ curve closely resembles that of the positron lifetime vs. time at $291 \mathrm{~K}$ with Al-Mg-Si alloys of similar Mg and Si concentrations (see the results of the alloys F of FIG. 6 in Ref. 6), in which approximate concentrations of solute $\mathrm{Mg}$ and $\mathrm{Si}$ are 0.67 at. $\%$ and 0.78 at.\%, respectively). The minimum of the lifetime for the alloy $\mathrm{F}$ was found at 50 minutes; this value is consistent with that extracted in Fig. 1.

Isothermal magnetization measurements with the Al-7-7 sample were carried out at 250, 270, 280, 290, 300 and $310 \mathrm{~K}$ in an external field of $7 \mathrm{~T}$. The time variations of $M$ were plotted in Fig. 2. Minima in $M$ are apparent in 280, 290 and $300 \mathrm{~K}$ data (marked by the arrows). Also there is a NA temperature dependence in the minimum time; a higher NA temperature leads to an earlier $M$ minimum time. This phenomenon is explained by the clustering processes of $\mathrm{Mg}$ - or/and $\mathrm{Si}$-vacancies. ${ }^{5,6)}$ The initial $M$ values at each individual NA temperature scattered around $0.0403 \mathrm{Am}^{2} \mathrm{~kg}^{-1}$ by about $1 \%$ in magnitude, most likely due to the diamagnetism of a kapton tape used for fixing the sample. This diamagnetism, however, hardly makes time dependent contributions to the magnetization.

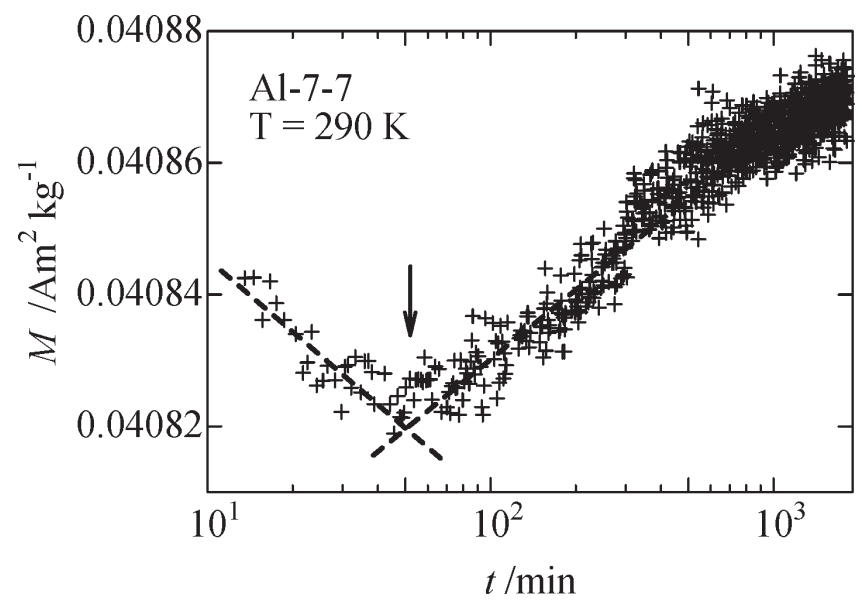

Fig. 1 Time dependence of magnetization of an Al-0.67 at. $\% \mathrm{Mg}-0.73$ at. $\% \mathrm{Si}$ alloy at $290 \mathrm{~K}$ and $7 \mathrm{~T}$. The time that the magnetization minimum occurs is marked by the arrow which in turn was determined as the intersection point of the two linear least-square fits.
Isothermal $M$ vs. $t$ with the Al-10-3 sample were measured at 270, 280, 290, 295, 300 and $320 \mathrm{~K}$ as shown in Fig. 3. A comparison of the results for the (Mg rich) Al-10-3 sample with those for the (Si rich) Al-7-7 reveals two distinct contributions of the solute concentrations to the $M$ variations with time. The $M$ minimum times at 280 and $290 \mathrm{~K}$ with the Al10-3 clearly occur later than those with the Al-7-7. The magnitudes of $M$ changes around $10^{3}$ minutes for the Al-10-3 are about double of those for the Al-7-7 above $290 \mathrm{~K}$; thus the solute $\mathrm{Mg}$ atoms seem to make positive contributions to magnetization in the clustering process in the later time ranges.

A measurement with the Al-10-5, additional to those of Ref. 25), was performed at $270 \mathrm{~K}$. The $M$ vs. $t$ curve is displayed in Fig. 4 together with our previous data sets at 280, 290 and $300 \mathrm{~K}$. Again the shift of $M$ minimum with NA temperature is obvious. The largest solute concentration of $\mathrm{Mg}+\mathrm{Si}$ among the three samples is considered to result in the largest $M$ value change of $1.6 \times 10^{-4} \mathrm{Am}^{2} \mathrm{~kg}^{-1}$ near $10^{3}$ minutes at $300 \mathrm{~K}$. The corresponding values for the Al-7-7 and Al-10-3 are $5.1 \times 10^{-4}$ and $9.2 \times 10^{-4} \mathrm{Am}^{2} \mathrm{~kg}^{-1}$, respectively.

The observed complex variations of $M$ vs. $t$ curves imply that the clustering processes of $\mathrm{Mg}$, Si and vacancy involves several stages with different magnitudes and time constants depending on the solute concentrations and the NA tempera-

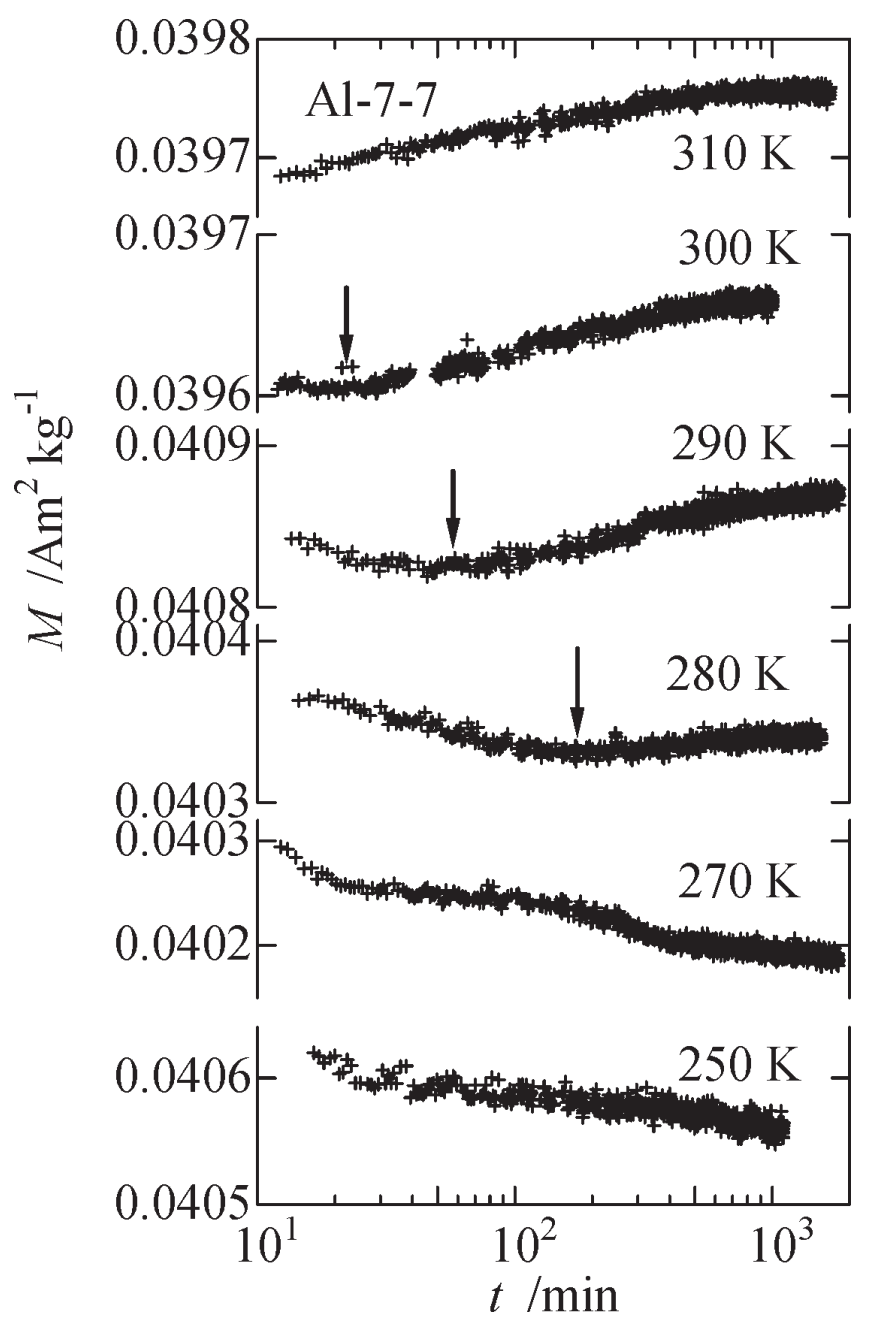

Fig. 2 Time dependence of magnetization of an Al-0.67 at.\%Mg-0.76 at. $\% \mathrm{Si}$ alloy at a constant temperature between 250 and $310 \mathrm{~K}$. 


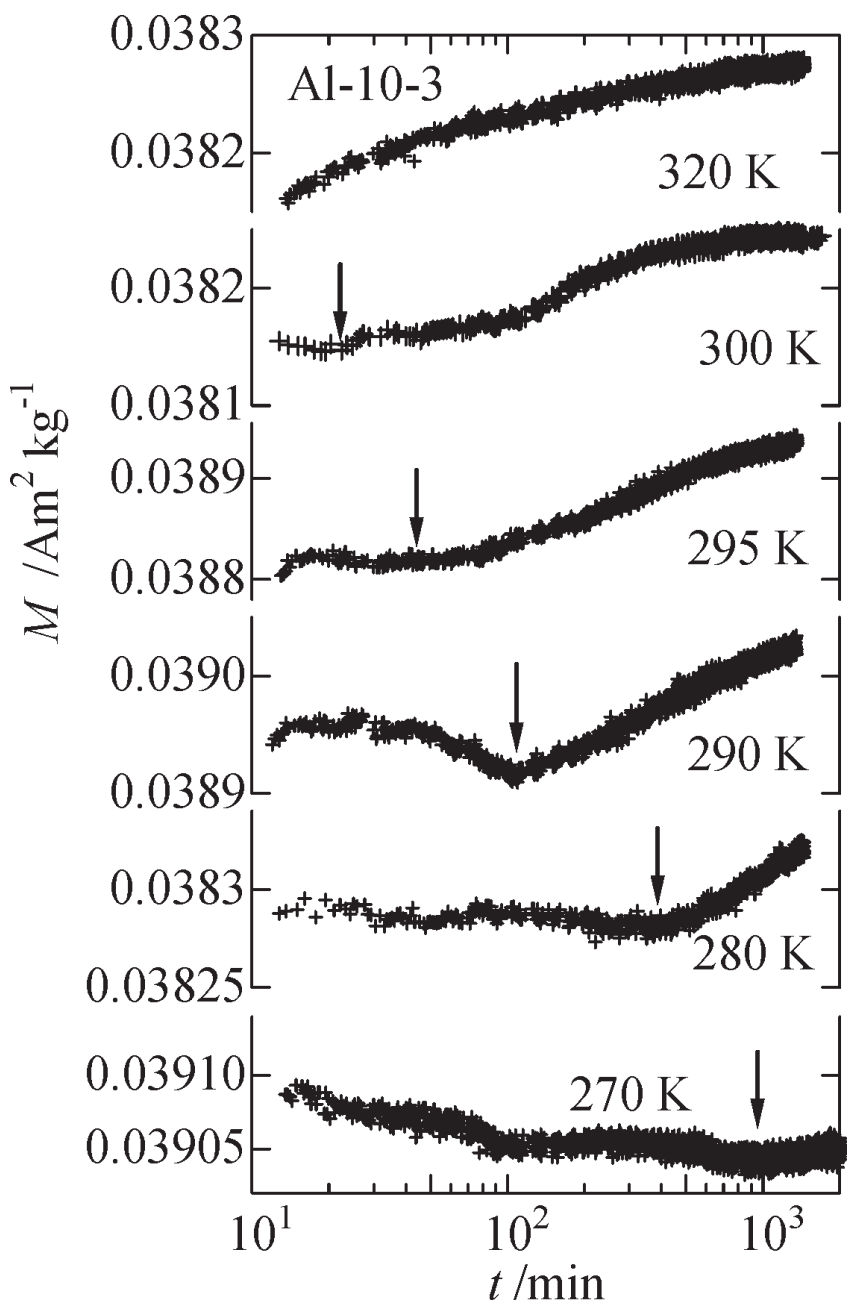

Fig. 3 Time dependence of magnetization of an Al-1.07 at.\%Mg-0.33 at. $\% \mathrm{Si}$ alloy at a constant temperature between 270 and $320 \mathrm{~K}$

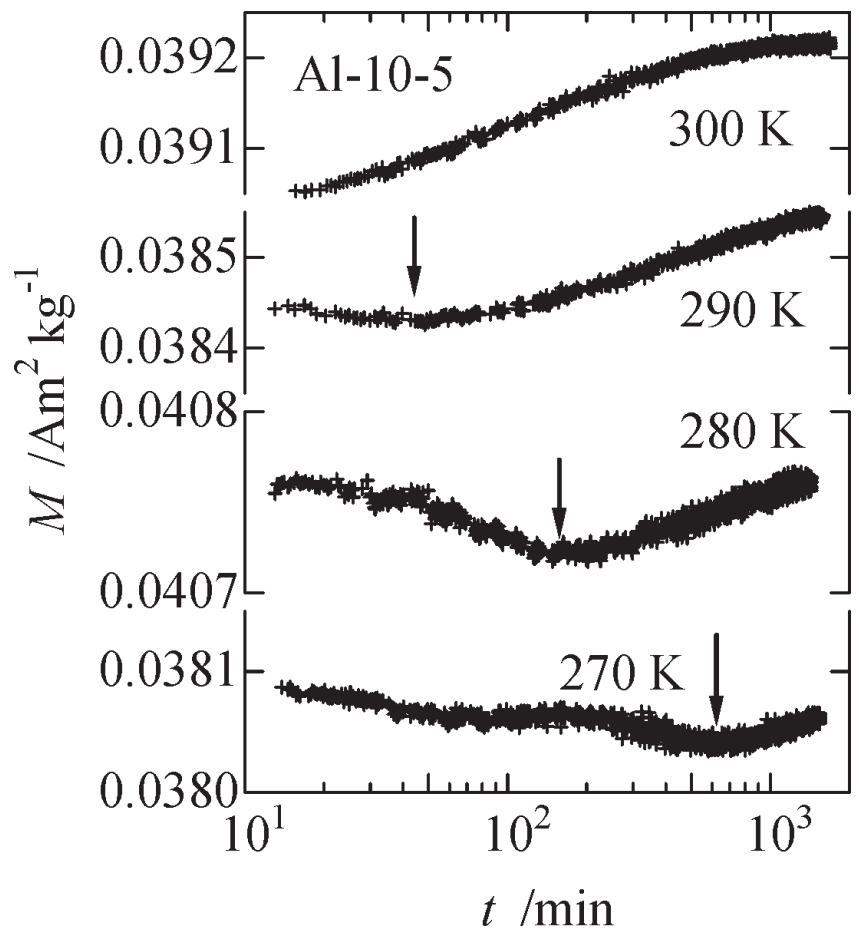

Fig. 4 Time dependence of magnetization of an Al-1.07 at.\%Mg-0.53 at. $\% \mathrm{Si}$ alloy at a constant temperature between 270 and $300 \mathrm{~K}$. (The data sets at 280, 290 and $300 \mathrm{~K}$ are quoted from Ref. 25))

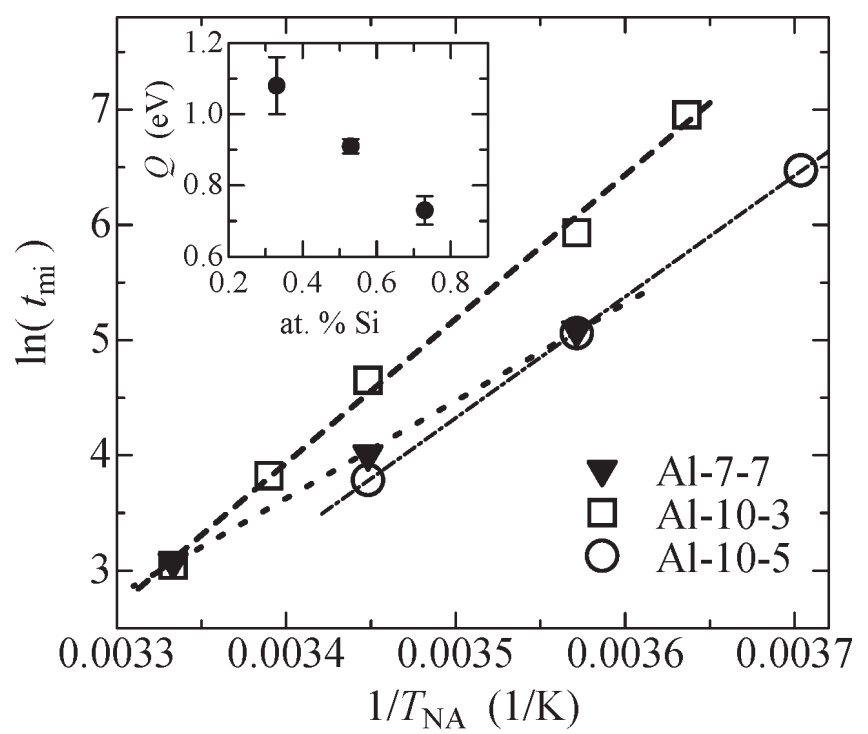

Fig. 5 Arrhenius plots for Al-0.67 at.\%Mg-0.73 at.\%Si, Al-1.07 at. $\% \mathrm{Mg}-0.33$ at. $\% \mathrm{Si}$ and $\mathrm{Al}-1.07$ at. $\% \mathrm{Mg}-0.53$ at. $\%$ Si using the magnetization minimum time and a natural aging temperature. The inset shows a plot of activation energy vs. Si concentration.

tures. While it is not possible to clarify cluster types from the present data, we have made an attempt to estimate the activation energy of clustering $(Q)$ following the methods used in the PAS study. ${ }^{6)}$ Since the observed $M$ minimum times $\left(t_{\min }\right)$ are well correlated to the transition times from stage II $(\mathrm{C} 1$, in which $\mathrm{Si}$ rich clusters form) to stage III (C2, Mg-Si co-clusters form) reported from the positron lifetime and resistivity studies under equivalent conditions, ${ }^{5,6)}$ we assume that the $t_{\text {min }}$ is related with the transition time from the stage II to III. In Fig. 5 we present Arrhenius plots of logarithmic $t_{\min }$ against reciprocal temperature of NA $\left(T_{\mathrm{NA}}\right), \ln \left(t_{\min }\right) \sim Q / k_{\mathrm{B}} T_{\mathrm{NA}}$, based on the data of Figs. 2, 3 and 4. Least square fits of the data yields $Q=0.73(+-0.04), 1.08(+-0.08)$ and 0.91 (+-0.02) eV for the Al-7-7, Al-10-3 and Al-10-5, respectively. The resulting stage II to III $Q$ values seem to be dependent on the sample Si concentrations; the $Q$ values increase with decreasing Si concentrations as shown in the inset in Fig. 5. This finding is consistent with the conclusion of the DSC studies, in which the DSC peaks for the $\mathrm{C} 1$ reaction shifted to higher temperatures with decreasing the $\mathrm{Si}$ concentrations of Al-Mg-Si alloys. ${ }^{19,21)}$ From this analysis it is suggested that the clustering process in the stage II is dominated by the action of the Si-rich clusters. The recent APT study by Zandbergen et al. ${ }^{17)}$ explains that relatively large size of $\mathrm{Mg}$ atoms favors formation of Si-rich clusters in an early time range due to relatively larger diffusivities of $\mathrm{Si}$. The deduced $Q$ value for the Al-7-7 agrees with that of $0.77(+-0.05) \mathrm{eV}$ for the samples F (Al-0.67 at.\% Mg-0.78 at.\%Si) in the PAS study. ${ }^{6}$ ) There are indeed several reports of $Q$ values in the range $0.32-0.90 \mathrm{eV}^{5,6,21)}$

There are two possible explanations for the observed $M$ vs. $t$ variations: $M$ values decrease during Si-rich clustering, but increase during $\mathrm{Mg}$-Si clustering. The Al-Mg-Si alloys are in the Pauli paramagnetic state, in which the magnetization is a linear function of the state density at the Fermi energy level and an external magnetic field. A recent soft X-ray absorption fine structure (XAFS) of an Al-Mg-Si alloy has reported that 
the valence of $\mathrm{Si}$ ions increased during the $\mathrm{Si}$-rich clustering, then decreased with NA time, while that of Mg ions gradually increased monotonically. ${ }^{26)}$ The valence changes of solute ions could lead to a change of the Fermi energy level, resulting in the change in the magnetization. It is also possible that the Si-rich clusters produce diamagnetic local magnetizations, but $\mathrm{Mg}$-Si clusters produce paramagnetic local magnetizations depending on their electronic structures. Unfortunately there is no relating experimental report, but a high intensity neutron scattering experiment could examine the local magnetism of Si-rich/ Mg-Si clusters.

\section{Conclusion}

This work has confirmed that a conventional DC magnetization measurement can be utilized to study the early stage clustering reactions in $\mathrm{Al}-\mathrm{Mg}-\mathrm{Si}$ alloys in isothermal conditions. The isothermal time variations of $M$ were found to depend on temperature as well as the solute $\mathrm{Mg}$ and Si concentrations. The observed $t_{\min }$ on $M$ vs. $t$ curve has temperature dependence similar to that of positron annihilation lifetime under equivalent conditions. Arrhenius plots of logarithmic $t_{\min }$ vs. reciprocal NA temperature provide activation energy from stage II to stage III, which are consistent with those in literature. In addition, the deduced values of the activation energy were found to depend on the solute Si concentrations, implying the predominant reactions of Si-vacancy in stage II. The present results of $M$ vs. $t$ curves would be useful to investigate forms of $\mathrm{Mg} / \mathrm{Si} /$ vacancy clusters in Al-Mg-Si alloys since the magnetization is closely related with the electronic structure of clusters.

\section{Acknowledgments}

One of the authors (K. Nishimura) acknowledges JSPS KAKENHI No.25289260 and Toyama Daiichi Bank Foundation.

\section{REFERENCES}

1) K. Matsuda, S. Taniguchi, K. Kido, Y. Uetani and S. Ikeno: Mater. Trans. 43 (2002) 2789-2795.

2) T. Moons, P. Ratchev, P.D. Smet, B. Verlinden and P.V. Houtte: Scr. Mater. 35 (1996) 939-945.
3) M. Murayama and K. Hono: Acta Mater. 47 (1999) 1537-1548.

4) S. Pogatscher, H. Antrekowitsch, H. Leitner, T. Ebner and P.J. Uggowitzer: Acta Mater. 59 (2011) 3352-3363.

5) H. Seyedrezai, D. Grebennikov, P. Mascher and H.S. Zurob: Mater. Sci. Eng. A 525 (2009) 186-191.

6) J. Banhart, M.D.H. Lay, C.S.T. Chang and A.J. Hill: Phys. Rev. B 83 (2011) 014101.

7) A. Somoza, A. Dupasquier, I.J. Polmear, P. Folegati and R. Ferragut: Phys. Rev. B 61 (2000) 14454-14463.

8) A. Dupasquier, G. Kogel and A. Somoza: Acta Mater. 52 (2004) 47074726.

9) M. Liu, J. Cizek, C.S.T. Chang and J. Banhart: Acta Mater. 91 (2015) 355-364.

10) F.A. Martinsen, F.J.H. Ehlers, M. Torsæter and R. Holmestad: Acta Mater. 60 (2012) 6091-6101.

11) C.D. Marioara, S.J. Andersen, J. Jansen and H.W. Zandbergen: Acta Mater. 51 (2003) 789-796.

12) S. Pogatscher, H. Antrekowitsch, M. Werinos, F. Moszner, S.S.A. Gerstl, M.F. Francis, W.A. Curtin, J.F. Löffler and P.J. Uggowitzer: Phys. Rev. Lett. 112 (2014) 225701.

13) K. Matsuda, H. Gamada, K. Fujii, Y. Uetani, T. Sato, A. Kamio and S. Ikeno: Metall. Mater. Trans., A Phys. Metall. Mater. Sci. 29 (1998) 1161-1167.

14) C.D. Marioara, H. Nordmark, S.J. Andersen and R. Holmestad: J. Mater. Sci. 41 (2006) 471-478.

15) A. Serizawa, S. Hirosawa and T. Sato: Matall. Mater. Trans A 39 (2008) 243-251.

16) M. Torsæter, H.S. Hasting, W. Lefebvre, C.D. Marioara, J.C. Walmsley, S.J. Andersen and R. Holmestad: J. Appl. Phys. 108 (2010) 073527.

17) M.W. Zandbergen, Q. Xu, A. Cerezo and G.D.W. Smith: Acta Mater. 101 (2015) 136-148.

18) A.K. Gupta and D.J. Lloyd: Metall. Mater. Trans. A30 (1999) 879-884

19) S. Kim, J. Kim, H. Tezuka, E. Kobayashi and T. Sato: Mater. Trans. 54 (2013) 297-303.

20) C.S.T. Chang and J. Banhart: Metall. Mater. Trans. A42 (2011) 19601964.

21) C. S. T. Changa, Z. Liang, E. Schmidt and J. Banhart: Int. J. Mat. Res. 103 (2012) 8-1-7.

22) S. Wenner, R. Holmestad, K. Matsuda, K. Nishimura, T. Matsuzaki, D. Tomono, F.L. Pratt and C.D. Marioara: Phys. Rev. B 86 (2012) 104201.

23) S. Wenner, K. Nishimura, K. Matsuda, T. Matsuzaki, D. Tomono, F.L. Pratt, C.D. Marioara and R. Holmestad: Acta Mater. 61 (2013) 6082 6092.

24) K. Nishimra, K. Matsuda, R. Komaki, N. Nunomura, S. Wenner, R. Holmestad, T. Matsuzaki, I. Watanabe, F.L. Pratt and C.D. Marioara: J. Phys. Conf. Ser. 551 (2014) 012031.

25) K. Nishimura, K. Matsuda, T. Namiki, N. Nunomra, T. Matsuzaki and W.D. Hutchison: Mater. Trans. 56 (2015) 1307-1309.

26) H. Adachi, H. Nakanishi, A. Hasegawa and M. Asano: Collected Abstracts of the 129th Conference of Japan Institute of Light Metals (2015) pp. 321-322. 